\title{
Estrategia de intervención nutricional a través del ciclo vital para la prevención de obesidad y otras enfermedades crónicas (EINCV) *
}

\author{
DIVISIÓN DE POLÍTICAS PÚBLICAS SALUDABLES Y DIVISIÓN INTEGRADA EN REDES. MINISTERIO \\ DE SALUD ${ }^{(1)}$
}

\section{INTRODUCCIÓN}

La Organización Mundial de la Salud ha propuesto una estrategia mundial sobre régimen alimentario, actividad física y salud, instando a los Estados participantes a implementarla. El desafío para los gobiernos es conseguir un cambio conductual de la sociedad y los individuos, que permita disminuir la obesidad y las enfermedades crónicas no transmisibles. Estas acciones además, deberán tener un criterio que corrija las inequidades relacionadas con la pobreza y el género.

En esta perspectiva, el Ministerio de Salud ha centrado su esfuerzo en la Estrategia Global contra la Obesidad (EGO-CHILE), para incluir e impulsar todas las iniciativas de carácter estructural e individual, que se vinculen con la promoción de estilos de vida saludables en la población. Su propósito fundamental es fomentar una alimentación saludable y el aumento de la actividad física a lo largo de todo el ciclo vital, con el fin, de contribuir a controlar la obesidad y las enfermedades crónicas asociadas. EGO es una invitación a las personas, a las instituciones públicas y privadas y a todos los sectores que quieran contribuir a un país más sano para un mejor desarrollo. Como parte de este esfuerzo, el año 2007 se inició una estrategia intersectorial, en los establecimientos educacionales (EGO Escuela), con miras a incor- porar desde lo curricular y extracurricular, el fomento de la vida sana.

En el marco de EGO-CHILE en la red asistencial, específicamente en la atención primaria de salud se desarrolla la Estrategia de Intervención Nutricional a través del Ciclo Vital (EINCV), que propone una visión integrada de la nutrición, alimentación y actividad física, en los diversos programas de salud.

La EINCV se enmarca también, en los Objetivos Sanitarios del país para la década y, en el contexto, de la prevención de enfermedades no transmisibles (ENT), especialmente las cardiovasculares, que se encuentran dentro de las prioridades país, por su gran impacto en la morbilidad y mortalidad de la población. El propósito principal es contribuir a la prevención de la obesidad y otras enfermedades crónicas relacionadas con la alimentación y la actividad física.

Con esta estrategia se pretende destacar ante los profesionales de salud y los beneficiarios del sistema, la magnitud de la actual epidemia de obesidad, sus enormes costos en términos de recursos y de calidad de vida, así como, la importancia que tiene el trabajo multidisciplinario e intersectorial, en la prevención y manejo del problema. Además, las acciones intersectoriales deben incorporar un trabajo con enfoque de los Determinantes Sociales en Salud (DSS), entendidas como las condiciones

\footnotetext{
(*) Extracto de las orientaciones en nutrición para la red asistencial, año 2008-2009 del Ministerio de Salud de Chile.

(1) Ministerio de Salud, Chile. Mac Iver 541, Santiago, Chile. 1rodriguez@minsal.cl
} 
sociales en las que viven y trabajan las personas o, como las características sociales dentro de las cuales la vida tiene lugar. Incluyen tanto las características específicas del contexto social que influyen en la salud, como las vías por las cuales las condiciones sociales en que la gente vive, se traducen en efectos en la salud.

Por otra parte, la evidencia científica sostiene que las acciones más costo efectivas son aquellas intervenciones iniciadas precozmente, especialmente en aquellas personas que desde las primeras etapas de la vida se pueden catalogar como de mayor vulnerabilidad. En este contexto, la EINCV aborda principalmente a niños, niñas, embarazadas y nodrizas. Adicionalmente, nuestro país está desarrollando un Sistema de Protección Integral a la Primera Infancia, Chile Crece Contigo (CHCC), dirigido a niñas y niños y a sus familias, de manera de apoyarlos para alcanzar su máximo potencial de desarrollo desde la gestación. CHCC tiene su expresión, en el ámbito del sistema de salud, en la generación de un programa que aborda el ámbito biológico, cognitivo y socio-emocional de la niña y el niño y la atención personalizada de la mujer embarazada.

\section{FUNDAMENTOS DE LA EINCV}

Las enfermedades crónicas (EC) relacionadas con la alimentación y la actividad física tales como: enfermedades cardiovasculares, diabetes tipo 2, obesidad y la osteoporosis entre otras, representan la principal causa de morbimortalidad en Chile. Según la Encuesta Nacional de Salud (ENS 2003) un 23\% de la población mayor de 17 años de edad presenta obesidad, mientras que un 38\% está en el rango de sobrepeso, factor de riesgo independiente para muchas enfermedades crónicas. Además, la reciente Encuesta de Calidad de Vida 2006 confirma que al menos un 90\% de la población califican como sedentarios, cifra que afecta mayormente a mujeres y al nivel socioeconómico bajo.

La obesidad "abdominal" diagnosticada por un perímetro de la cintura aumentado, constituye una causa importante de hiperinsulinemia, resistencia a la insulina, intolerancia a glucosa, diabetes tipo 2, hipertensión arterial, dislipidemia aterogénica (hipertrigliceridemia, aumento de colesterol LDL y disminución de colesterol HDL) y disfunción endotelial (por la acción de factores protrombóticos y proinflamatorios). La presencia de obesidad abdominal y dos o más de las alteraciones anteriores, constituyen un "Síndrome Metabólico" (SM), presente en uno de cada 4 adultos chilenos. Ello se refleja en una alta prevalencia de hipertensión arterial (35\%), hipercolesterolemia (35\%), hipertrigliceridemia $(30 \%)$, intolerancia a la glucosa $(16 \%)$ y diabetes $(4 \%)$, todas las cuales presentan un aumento importante en las personas de mayor edad. Con estos datos el $50 \%$ de los adultos chilenos presentan un riesgo cardiovascular alto o muy alto.

En la población infantil, la obesidad se acompaña también de un aumento de sus complicaciones médicas. La obesidad abdominal, la resistencia insulínica (RI) y el antecedente familiar de enfermedades crónicas, son las variables más asociadas al riesgo de síndrome metabólico (SM) en los niños. En Chile, el SM afecta al 30\% de los niños obesos (IMC $\geq$ p 95) y al 4,7\% de los niños con sobrepeso (IMC entre p 85 y 95).

Para evaluar la implementación de la EINCV el año 2005 y 2006 se efectuó un seguimiento de las embarazadas y postparto de dos cohortes; una con la modalidad tradicional de atención (sin EINCV) y la segunda que recibió la nueva propuesta de atención (con EINCV). Entre los resultados, aún no publicados, destacan diferencias entre ambos grupos al analizar la situación nutricional de la mujer al $6^{\circ}$ mes post parto; las mujeres con obesidad intervenidas con la nueva metodología (con EINCV), tienden a recuperan más su peso pregestacional que aquellas intervenidas con metodología tradicional $(\mathrm{p}<0,005)$. Un hallazgo interesante fue descubrir la clara interacción entre el estado nutricional al inicio del embarazo y haber pertenecido al grupo de la EINCV; así, las mujeres que inician su embara- 
zo con obesidad reciben una atención focalizada, por los profesionales de los establecimientos de salud, contribuyendo a que, no tan sólo recuperen su peso pregestacional, sino que se acerquen a una situación nutricional normal.

\section{ESTADO NUTRICIONAL DE POBLACIÓN MATERNO INFANTIL}

LA

La situación nutricional de las embarazadas controladas en la red asistencial del sistema público de salud se presenta en la Tabla 1. Destaca que más de la mitad de las mujeres en control presenta un exceso de peso, y que existe una reducida proporción de bajo peso. Como en otros grupos poblacionales, hay un aumento significativo de la

Tabla 1. Prevalencia de estado nutricional de la embarazada según grupo de edad durante los años 2005 - 2007.

\begin{tabular}{|c|c|c|c|}
\hline & $\begin{array}{c}2005 \\
\% \\
\end{array}$ & $\begin{array}{c}2006 \\
\% \\
\end{array}$ & $\begin{array}{c}2007 \\
\% \\
\end{array}$ \\
\hline \multicolumn{4}{|l|}{ Bajo peso } \\
\hline$<20$ & 13,7 & 14,1 & 14,0 \\
\hline $20-34$ & 6,6 & 6,4 & 6,1 \\
\hline$\geq 35$ & 2,4 & 2,8 & 2,5 \\
\hline Total & 7,7 & 7,7 & 7,5 \\
\hline \multicolumn{4}{|l|}{ Sobrepeso } \\
\hline$<20$ & 24,0 & 23,9 & 24,4 \\
\hline $20-34$ & 32,5 & 33,0 & 32,6 \\
\hline$\geq 35$ & 37,8 & 38,3 & 38,9 \\
\hline Total & 31,2 & 31,6 & 31,5 \\
\hline \multicolumn{4}{|l|}{ Obesas } \\
\hline$<20$ & 9,8 & 9,1 & 9,2 \\
\hline $20-34$ & 21,7 & 20,8 & 21,7 \\
\hline$\geq 35$ & 32,3 & 31,6 & 31,4 \\
\hline Total & 20,3 & 19,4 & 20,0 \\
\hline
\end{tabular}

Fuente: Departamento de estadística e información de salud, Ministerio de Salud obesidad a medida que aumenta la edad, que es tres veces mayor en las mayores de 35 años respecto a las adolescentes. Inversamente, se produce una fuerte disminución del bajo peso, reforzando la idea de que el ciclo reproductivo puede contribuir a aumentar el riesgo de sobrepeso. La comparación entre los tres últimos años no muestra cambios importantes.

Un análisis similar a los 6 meses post parto se presenta en la Tabla 2. Entre el 2005 y 2007 aumentó en forma importante el número de mujeres evaluadas, reflejando un mejor cumplimiento de la norma. También, destaca una reducción de la prevalencia de obesidad al 2007, en todas las edades, sugiriendo un impacto positivo de la estrategia de intervención.

Tabla 2. Prevalencia de estado nutricional de la mujer a los 6 meses post parto según grupo de edad durante los años $2005-2007$.

\begin{tabular}{|c|c|c|c|}
\hline & $\begin{array}{c}2005 \\
\mathrm{n}=13.843 \\
\%\end{array}$ & $\begin{array}{c}2006 \\
\mathrm{n}=20.089 \\
\%\end{array}$ & $\begin{array}{c}2007 \\
\mathrm{n}=42.734 \\
\%\end{array}$ \\
\hline \multicolumn{4}{|l|}{ Bajo peso } \\
\hline$<20$ (años) & 9,9 & 8,1 & 7,3 \\
\hline $20-34$ & 4,6 & 4,0 & 3,2 \\
\hline$\geq 35$ & 2,1 & 1,9 & 1,4 \\
\hline Total & 5,4 & 4,6 & 3,9 \\
\hline \multicolumn{4}{|l|}{ Sobrepeso } \\
\hline$<20$ (años) & 28,1 & 30,0 & 27,1 \\
\hline $20-34$ & 33,5 & 35,1 & 34,0 \\
\hline$\geq 35$ & 35,6 & 39,8 & 40,1 \\
\hline Total & 32,6 & 34,6 & 33,2 \\
\hline \multicolumn{4}{|l|}{ Obesas } \\
\hline$<20$ (años) & 15,2 & 13,8 & 12,7 \\
\hline $20-34$ & 26,6 & 23,6 & 22,3 \\
\hline$\geq 35$ & 37,9 & 32,7 & 31,6 \\
\hline Total & 25,7 & 22,6 & 21,3 \\
\hline
\end{tabular}

Fuente: Departamento de estadística e información de salud, Ministerio de Salud 
La comparación del estado nutricional de la embarazada y a los 6 meses post parto se presenta en la Tabla 3. Aunque, los criterios diagnósticos no son estrictamente comparables, se observa una reducción del bajo peso a la mitad y un discreto aumento del sobrepeso y la obesidad a los 6 meses post parto, reforzando la importancia de evitar aumentos excesivos de peso durante la gestación.

Tabla 3. Comparación de la prevalencia del estado nutricional durante el embarazo con los 6 meses post parto en el año 2007.

\begin{tabular}{|c|c|c|}
\hline & $\begin{array}{c}\text { Embarazadas } \\
\%\end{array}$ & $\begin{array}{c}6 \text { meses } \\
\text { post parto } \\
\%\end{array}$ \\
\hline \multicolumn{3}{|l|}{ Bajo peso } \\
\hline$<20$ & 14,0 & 7,3 \\
\hline $20-34$ & 6,1 & 3,2 \\
\hline$\geq 35$ & 2,5 & 1,4 \\
\hline Total & 7,5 & 3,9 \\
\hline \multicolumn{3}{|l|}{ Sobrepeso } \\
\hline$<20$ & 24,4 & 27,1 \\
\hline $20-34$ & 32,6 & 34,0 \\
\hline$\geq 35$ & 38,9 & 40,1 \\
\hline Total & 31,5 & 33,2 \\
\hline \multicolumn{3}{|l|}{ Obesa } \\
\hline$<20$ & 9,2 & 12,7 \\
\hline $20-34$ & 21,7 & 22,3 \\
\hline$\geq 35$ & 31,4 & 31,6 \\
\hline Total & 20,0 & 21,3 \\
\hline
\end{tabular}

Fuente: Departamento de estadística e información de salud, Ministerio de Salud

En cuanto a los menores de 6 años destaca una muy baja prevalencia de déficit nutricional; sólo el 2,1 \% de la población en control tiene una relación talla-edad $<$ de $2 \mathrm{DE}$ en relación a la re- ferencia OMS, que es la proporción esperada en la distribución normal. Sin embargo, existe una alta prevalencia de sobrepeso y obesidad, especialmente a partir de los 4 años de edad (Tabla 4). Según lo esperado, la nueva referencia OMS determinó un aumento de la obesidad en dos puntos porcentuales y una diferencia aún mayor entre 1 y 3 años de edad. La actual prevalencia obtenida con la referencia OMS deberá usarse como línea de base para evaluar los impactos futuros.

Tabla 4. Prevalencia de sobrepeso y obesidad por grupo de edad, en Chile durante los años 2006 - 2007.

\begin{tabular}{|l|l|l|}
\hline Relación peso-talla & $\begin{array}{l}\mathbf{2 0 0 6} \\
\mathbf{\%}\end{array}$ & $\begin{array}{l}\mathbf{2 0 0 7} \\
\mathbf{\%}\end{array}$ \\
\hline $\begin{array}{l}\text { Mayor igual 1 DE } \\
\text { (incluye también > 2 DE) }\end{array}$ & & \\
\hline $0-5$ meses & 20,9 & 21,2 \\
\hline $6-11$ meses & 27,4 & 32,2 \\
\hline $12-23$ meses & 22,8 & 34,2 \\
\hline $24-47$ meses & 20,5 & 31,1 \\
\hline $48-71$ meses & 29,8 & 33,6 \\
\hline Total & $\mathbf{2 4 , 5}$ & $\mathbf{3 1 , 7}$ \\
\hline Mayor igual 2 DE & \multicolumn{2}{|l|}{} \\
\hline $0-5$ meses & 5,0 & 5,3 \\
\hline $6-11$ meses & 7,3 & 8,8 \\
\hline $12-23$ meses & 6,2 & 9,2 \\
\hline $24-47$ meses & 5,7 & 9,2 \\
\hline $48-71$ meses & 11,7 & 12,2 \\
\hline Total & $\mathbf{7 , 8}$ & $\mathbf{9 , 8}$ \\
\hline
\end{tabular}

Fuente: Departamento de estadística e información de salud, Ministerio de Salud

\section{ROL DE LA ATENCIÓN PRIMARIA}

Los equipos de salud tienen un importante rol, en la orientación hacia las madres, padres, cuidadoras(es), adolescentes, niñas(os) y la comunidad en general, desde temprana edad, para promover estilos de vida que prevengan problemas de obesidad. En este contexto, se releva la consejería como una relación de acercamiento entre el profesional de salud y un usuario, que 
pretende estimular el cambio de comportamiento favorable. Se pretende que, a través de la consejería, se fomente la capacidad del individuo para tomar decisiones con respecto a su salud y actuar en consecuencia. Esta metodología implica un proceso a través del cual, el profesional de la salud puede ayudar al usuario a tomar decisiones favorables para su salud y bienestar. La efectividad de la consejería ha sido demostrada tanto, para el cambio en hábitos alimentarios como de actividad física. En estas actividades grupales o individuales, hay que hacer énfasis en el fomento de los factores protectores, en especial el fortalecimiento familiar, el refuerzo del vínculo escolar y la existencia y participación en redes sociales.

\section{INTERVENCIONES EN LA MUJER}

El análisis de la situación nutricional de los adultos en nuestro país, revela que predominan los problemas de malnutrición por exceso en especial en la mujer y en los grupos de menores ingresos económicos. Se requiere entonces, reforzar las estrategias de intervención nutricional con miras a prevenir los potenciales efectos sobre la salud y el pronóstico materno-infantil, a corto y largo plazo.

La evidencia médica disponible, fundamenta la importancia de educar a las mujeres respecto de una alimentación y un estilo de vida sano, antes y durante el periodo de gestación.

El período preconcepcional es de vital importancia en términos de preparación para la etapa de gestación. Es decir, para que la mujer logre condiciones óptimas para el desarrollo de un buen embarazo, ya sea; corrigiendo su situación nutricional, controlando patologías como diabetes o hipertensión, suspendiendo el hábito tabáquico, entre otros. En el embarazo, el período más crítico son las primeras ocho a doce semanas, cuando recién se inician los mecanismos de formación de la placenta; en ese momento, intervienen en forma negativa las condiciones mórbidas descritas, cuando aún, en muchos casos, la madre no sabe que está embarazada.
El ambiente nutricional intrauterino releva su importancia en términos de imprimir en el feto las características metabólicas que influyen sobre su riesgo de desarrollar obesidad o enfermedades crónicas durante la vida adulta. En este contexto, existe evidencia formulada en la última década sobre el "origen fetal" de las enfermedades crónicas del adulto, aportada originalmente de las pioneras observaciones del epidemiólogo inglés David Barker. Estas resaltan el rol de la nutrición materna durante la gestación y, por ende, el potencial valor de la evaluación y consejería nutricional en este período; con una mirada de la prevención de efectos adversos en la vida adulta. Así, en el periodo gestacional, la obesidad constituye un riesgo obstétrico y, además, representa para el niño(a) un riesgo de macrosomía y eventual malnutrición por exceso en su vida adulta.

Considerando las variables descritas, el Programa Nacional de Alimentación Complementaria del Minsal (PNAC), ha desarrollado una bebida láctea, "Purita Mamá", especialmente destinada a mujeres embarazadas y en lactancia. Este producto es de menor contenido de grasa y lactosa que la tradicional Leche Purita Fortificada y además, está fortificado con vitaminas y minerales. Su principal ventaja es que se presenta adicionada con de ácidos grasos omega-3 de cadena larga, específicamente ácido eicosapentaenoico (EPA) y ácido docosahexaenoico (DHA), nutrientes esenciales para el desarrollo cerebral y retiniano del feto y del recién nacido. Diversos estudios, han demostrado que un mayor aporte de DHA en el embarazo y en las fórmulas infantiles, reduce la proporción de partos prematuros y de recién nacidos con restricción del crecimiento intrauterino, además, mejora la agudeza visual y el desarrollo psicomotor del niño, aún hasta los 4 años de vida. Chile es pionero en el mundo en este tipo de programas.

En el postparto, un elemento importante a cuidar es la recuperación del peso pregestacional en la mujer. La prevalencia de sobrepeso en embarazadas es $31,5 \% ; 20 \%$ de obesidad y al $6^{\circ}$ mes post parto 21,3\% (DEIS Minsal, 2007). Por 
tanto, es evidente la necesidad de actuar sobre este problema, con el fin de evitar la retención del exceso de peso postparto.

\section{INTERVENCIONES EN LA NIÑA(O)}

En la mirada de prevención precoz, es útil considerar algunos factores que constituyen riesgo de EC:

- Obesidad en la madre o el padre

- Diabetes Mellitus tipo 2 en padre, madre o abuelos

- Diabetes gestacional

- Sobrepeso u obesidad gestacional

- Bajo peso de nacimiento (desnutrición intrauterina)

- Macrosomía del recién nacido (peso mayor a $4 \mathrm{Kg}$.)

- Lactancia materna exclusiva de insuficiente duración ( $<$ 4-6 meses)

- Incremento acelerado de peso durante la infancia (cruce de percentiles ascendentes) u obesidad

Áreas relevantes de intervención, por su potencial relación con los orígenes de la actual epidemia de obesidad, son la promoción de la lactancia materna y de prácticas adecuadas de alimentación en el período de destete. La evidencia es consistente en señalar los beneficios de la lactancia materna exclusiva y su efecto protector en términos de desarrollo de obesidad y otras EC; de allí, la importancia de reforzar las actuales actividades de promoción de la lactancia materna idealmente, exclusiva hasta los 6 meses, y óptimamente, prolongarla hasta el año de vida, junto a un régimen de alimentación mixta (leche materna y alimentos sólidos).

Las preferencias alimentarías se aprenden muy temprano en la niñez, en la etapa de incorporación del niño(a) a la alimentación familiar. Estas preferencias alimentarias condicionarán los hábitos de alimentación a edades posteriores.
Los cuidadores y el grupo familiar deben estar preparados, tanto para apoyar el aprendizaje, como para preservar la capacidad innata del niño y niña de alimentarse según sus requerimientos, obedeciendo a señales internas que lo llevan a mantener un adecuado balance energético.

La restricción absoluta de algunos alimentos; el uso de alimentos como recompensa a conductas deseadas; el condicionar la entrega de alimentos que gustan más (como por ejemplo el postre) a la ingesta previa de otro alimento que no prefiere (como algunas verduras o pescado); el obligar al niño(a) a tomarse hasta la última gota de la mamadera o a "limpiar el plato"; y otros patrones culturales que se dan en torno a la alimentación en esta etapa crítica, deben corregirse a través de un gran esfuerzo informativo, educacional y motivacional tanto de los equipos de salud como de la comunidad, incentivando, por ejemplo, la participación en grupos de pares o de autoayuda.

\section{PRINCIPALES OBJETIVOS DE LA EINCV}

1. Intervenir precozmente a la población infantil y a las mujeres embarazadas, idealmente desde la etapa preconcepcional, con la promoción de alimentación sana y actividad física periódica, a través de los controles de salud; haciendo énfasis en la población con mayor riesgo de desarrollar obesidad y enfermedades crónicas (EC), según la evidencia disponible.

2. Vigilar el estado nutricional y el incremento de peso durante el embarazo, interviniendo oportunamente en las desviaciones de la normalidad.

3. Controlar la recuperación del peso pregestacional en la mujer postparto.

4. Promover la lactancia materna exclusiva hasta el $6^{\circ}$ mes de vida.

5. Vigilar el estado nutricional y el incremento 
de peso durante la infancia e intervenir oportunamente en las desviaciones de la normalidad.

\section{ESTRATEGIA OPERATIVA DE LA EINCV}

La EINCV consiste en coordinar las actividades de promoción de salud que desarrollan los diferentes programas matriciales. Esta coordinación permitirá actuar de modo integral sobre los grupos familiares en riesgo.

Las acciones comprendidas en esta estrategia son canalizadas en la red asistencial en la atención primaria a través de, los Programas de Salud de la Mujer y de la Niña(o), siendo complementadas con las ya existentes en el Programa de Enfermedades Cardiovasculares, Programa Odontológico, Programa del Adulto Mayor y otros. De igual manera, se reforzarán con las acciones del Plan Nacional y de los Planes Comunales de Promoción, del Sistema Chile Crece Contigo y de la Estrategia EGO-Chile.

\section{EJES DE INTERVENCIÓN DE LA EINCV}

1. Relevar el tema nutricional a nivel de equipo de salud y población. se refuerza la motivación y capacitación del equipo de salud, en la aplicación de la EINCV, a través, de las diferentes prestaciones de salud que se desarrollan en los Establecimientos de la Red de Atención Primaria de Salud (APS), incluyendo algún tipo de consejería en alimentación saludable y actividad física, con focalización en los grupos de mayor riesgo detectados en la vigilancia nutricional.

2. Incorporar la consejería en alimentación saludable y actividad física en todos los controles habituales de la mujer y de la niña(o). La base de los contenidos entregados corresponden a las Guías Alimentarias, Guías para una Vida Activa y Guías de Vida Sana; el énfasis debe estar en favorecer la disminu- ción del consumo de las grasas trans y saturadas, reducción de azúcares simples y sodio; además, debe promoverse el consumo de verduras, frutas y leguminosas para mejorar el aporte de potasio y fibra. Junto a ello, estimular la actividad física y disminuir las horas de inactividad (horas de TV, computación). Para conseguir estos cambios, se requiere indagar sobre hábitos del paciente, referidos entre otros, a tiempos de comida, tipos de alimentos consumidos, frecuencia y volumen de los mismos, así como hábitos de ejercicio. Lo anterior, será de gran utilidad para negociar pequeños cambios y compromisos a cumplir, los que, serán específicos e individuales.

3. Cautelar el aumento de peso excesivo durante el embarazo. La vigilancia de la ganancia de peso durante el embarazo y en especial de aquellos casos que comienzan la gestación con sobrepeso u obesidad y aquellos que tienen antecedentes de factores de riesgo de EC, deberá realizarse estrechamente, procurando no sobrepasar las metas normadas según peso inicial, controlar la ingesta excesiva, al mismo tiempo cubrir los requerimientos específicos de ciertos micronutrientes y mantener un grado de actividad física saludable. En este contexto, la incorporación de la "Purita Mamá" contribuye a triplicar el consumo de DHA en la mujer embarazada y nodriza, permitiendo disminuir la brecha nutricional y de equidad en un nutriente esencial para el desarrollo cerebral y visual del niño(a). Esta entrega está ligada a la condición nutricional de la mujer al inicio del embarazo y a la condición de lactancia materna.

4. Promover la recuperación del estado nutricional pregestacional. Durante los primeros 6 meses post-parto se efectúa una vigilancia, procurando que la mujer retorne al menos a su peso pregestacional, e idealmente continúe su baja hasta una condición cercana a la normalidad. Para ello se realizan, al 
menos, dos controles exclusivos de la mujer, al 3er mes y 6to mes postparto, en los que se evalúa situación nutricional y se educa en alimentación saludable y actividad física. Es importante favorecer su vinculación a otros programas sectoriales, tales como el de Chiledeporte u otros comunales.

5. Promover lactancia materna. Esto significa que desde el periodo prenatal y desde los primeros controles de salud del niño y niña debe apoyarse a la mujer a consolidar una lactancia exitosa. Contar con clínicas de lactancia materna ha resultado eficaz en la detección oportuna de problemas y dudas que interfieren con la duración de la lactancia materna. La evidencia es consistente en demostrar sus beneficios en términos de protección de la obesidad y otras EC, por lo que, no se deben escatimar esfuerzos en este sentido.

6. Intervenir oportunamente en la infancia

a) En el Programa de Salud del Niño(a) se contempla una consulta nutricional al 5to mes de vida con el objeto de reforzar la lactancia materna exclusiva y educar oportunamente en alimentación al destete, procurando prevenir el desarrollo de malos hábitos alimentarios. Especial atención debe tenerse en este periodo, por las neofobias (aversión a la incorporación de nuevos sabores) que deben manejarse según las referencias en las Guías Alimentarias, evitando creencias que puedan establecer conductas afines a unos u otros sabores o alimentos. Se requiere de educación a las familias en el manejo de problemas frecuentes en la alimentación de los primeros años de vida y derribar mitos en relación a la nutrición.

b) Además, está indicada una consulta nutricional a los $3 \frac{1}{2}$ años de vida con componente de salud bucal, para todos las niñas y niños, para reforzar educación en alimentación, detectar y corregir conductas poco saludables e incorporar hábitos de higiene y salud bucal.Además, esta consulta debe preparar el ingreso de las niñas y niños al mundo educacional, orientando por ejemplo, en colaciones saludables, horarios y contenidos de las comidas.

c) Para las niñas y niños menores de 6 años, se cuenta con una "Norma de Manejo del Niño(a) con Malnutrición" que provee un flujograma de actividades individuales y grupales para enfrentar las desviaciones de la normalidad, el déficit, el exceso y los riesgos.

d) Los escolares y adolescentes que se detecten con problemas de malnutrición deben ser derivados a consulta nutricional e idealmente, al Programa de Alimentación Saludable y Actividad Física para Niñas, Niños y Adolescentes (PASAF niñas, niños y adolescentes).

7. Aprovechar redes de apoyo. Las redes están constituidas por la propia red asistencial de salud y especialmente en este ámbito la atención primaria de salud, los grupos y organizaciones comunales, el Consejo de Desarrollo Local (CESFAM - CECOF), establecimientos educacionales, JUNJ, JUNAEB, INTEGRA, Chiledeportes, entre otros, con los cuales, debe establecerse un vínculo cercano que permita desarrollar tareas conjuntas en pro de favorecer la alimentación saludable, la actividad física y la vida saludable.

\section{SEGUIMIENTO DE LA EINCV}

El seguimiento de esta estrategia debe considerar el acompañamiento por parte de los equipos de salud de la atención primaria en su quehacer, reforzando la capacitación, los programas 
ya existentes y vigilando a través del Departamento de estadística e información de salud la evolución de ciertos indicadores muy ligados a los Objetivos Sanitarios y a los ejes principales de la EINCV.

El sistema de salud chileno nos ofrece una gran oportunidad de intervenir precozmente además, de detectar factores de riesgo entre los grupos de población, que tradicionalmente han estado cubiertos desde la atención primaria y que están cautivos en sus controles ligados a vacunación y programas alimentarios. La consejería como pilar de esta estrategia abre la posibilidad de generar cambios de conducta en el seno de la familia.

Sin duda, que la EINCV debe estar complementada con programas y políticas de salud pública que aborden determinantes sociales y estructurales, necesarios para la construcción de una sociedad más saludable.

\section{REFERENCIAS}

1. REECE A. Perspectives on obesity, pregnancy and birth outcomes in the United States: The scope of the problem. American Journal of Obstetrics and Gynecology, Volume 198, Issue 1, Pages 23-27. 2008

2. MINISTERIO DE SALUD. GOBIERNO DE CHILE. "Norma para el manejo ambulatorio de la Malnutrición por déficit y exceso en el niño(a) menor de 6 años". 2007.

3. MINISTERIO DE SALUD. GOBIERNO DE CHILE. Departamento de Epidemiología y Departamento de Promoción y Participación Ciudadana. "II Encuesta Nacional de Calidad de Vida y Salud Chile". 2006.

4. MINISTERIO DE SALUD. GOBIERNO DE CHILE, "Estrategia Global Contra la Obesidad, EGO Chile". 2006.

5. MINISTERIO DE SANIDAD Y CONSUMO, AGENCIA ESPAÑOLA DE SEGURIDAD ALIMENTARIA. "Estrategia para la nutrición, actividad física y prevención de la obesidad". (NAOS). España 2005.

6. ATALAH E, CASTRO R. "Obesidad materna y riesgo reproductivo”. Rev Méd Chile. 132: 923 -
930. Chile 2004.

7. NUTRISCIENCE. "Ácidos grasos poliinsaturados de cadena larga en la nutrición materna". 2004.

8. MINISTERIO DE SALUD. GOBIERNO DE CHILE. Departamento de Epidemiología. "Encuesta Nacional de Salud". 2003.

9. MORENO LA, PINEDA I, RODRIGUEZ G, FLETA J, SARRÍA A, BUENO M. "Waist circumference for the screening of the metabolic syndrome in children". Acta Paediatr, 91: 130712. 2002.

10. DIET, NUTRITION AND THE PREVENTION OF CHRONIC DISEASES - Report of the Joint WHO/FAO expert consultation" (TRS 916). 2002.

11. R. ARTAL, M. O. TOOLE: "Guidelines of the American College of Obstetricians and Gynecologists for exercise during pregnancy and postpartum period". www.bjsportmed.enero. 2002.

12. SINGHAL A. ET AL. Early Origins of Obesity. En "Obesity in Childhood and Adolescence". Editores: Ch. Chen y W. H. Dietz. Nestlé Nutrition Workshop Series. Pediatric Program, Volume 49. 2002

13. WILLIAMS C. L. ET AL. "Cardiovascular Health in Childhood. Circulation" 106: 143. 2002.

14. L. L. BIRCH: Childhood Overweight: Family Environmental Factors. En "Obesity in Childhood and Adolescence". Editores: Ch. Chen y W. H. Dietz. Nestlé Nutrition Workshop Series. Pediatric Program, Volume 49. 2002.

15. AROS S, CASSORLAF. Posibles "Determinantes perinatales de morbilidad en la edad adulta". Rev. Méd. 129: 307-315. Chile 2001.

16. CASTILlO C.; ATALAH E.; UAUY R. "Guías de Alimentación para la Mujer". R. Burrows; Diario La Nación. Chile 2001.

17. DIETZ W. Health "Consequences of obesity in youth: Childhood predictors of adult disease. Pediatrics". 108 (3 II): 518S-25S. 1998.

18. M. GORAN: Obesity and Health Risk in Children. "Childhood Obesity: Causes and Prevention". Symposium Proceedings. Center for Nutrition Policy and Promotion. USDA, Washington D.C. 1998

19. BARKER DJP "Fetal nutrition and cardiovascular disease in the adult". Lancet 341:938-941. 1993. 
20. I.N.T.A. INSTITUTO DE NUTRICIÓN Y TECNOLOGÍA DE LOS ALIMENTOS. "Manual de Apoyo Consejerías en vida Sana".
21. GOBIERNO DE CHILE, http://www.crececontigo.cl/

Usted puede comentar éste y otros artículos publicados en la Revista Chilena de Salud Pública, enviando un correo electrónico a revistasp@med.uchile.cl 